Original Paper http://ajol.info/index.php/ijbcs http://indexmedicus.afro.who.int

\title{
Des pratiques culturales influent sur les attaques de deux ravageurs de la tomate dans les Niayes au Sénégal
}

\author{
Mamadou DIATTE ${ }^{1,2^{*}}$, Thierry BRÉVAULT ${ }^{3,4}$, Diénaba SALL-SY ${ }^{2}$ \\ et Karamoko DIARRA ${ }^{1}$ \\ ${ }^{1} U C A D$, Equipe Production et Protection Intégrées en Agro écosystèmes Horticoles - 2PIA, \\ Faculté des Sciences et Techniques, Dakar, Sénégal. \\ ${ }^{2}$ ISRA, Centre pour le Développement Horticole, CDH, Dakar, Sénégal. \\ ${ }^{3}$ BIOPASS, ISRA-UCAD-IRD, Dakar, Senegal. \\ ${ }^{4}$ CIRAD, UPR AIDA, F-34398 Montpellier, France. \\ *Auteur correspondant ; E-mail: mamadou.diatte@hotmail.fr; Tel.: +221 772324996
}

\section{REMERCIEMENTS}

Nous exprimons nos sincères gratitudes à l'AIRD (PEERS-BIOBIO) et au programme de production agricole en Afrique de l'Ouest (PPAAO/WAAPP) pour avoir soutenu financièrement cette étude.

\section{RESUME}

La culture de tomate est attaquée par plusieurs ravageurs dont Helicoverpa armigera et Tuta absoluta. Dans le but d'évaluer l'effet des pratiques culturales de la tomate sur ces principaux ravageurs dans les Niayes (Sénégal), un échantillonnage de 98 parcelles est effectué, sur quatre cycles de culture en saison sèche, de 2012 à 2014. Les pratiques culturales sont recueillies par questionnaire lors des visites auprès des producteurs. Les résultats ont montré que l'incidence de $H$. armigera et de $T$. absoluta est négativement corrélée à la distance à la mer. La densité des plants dans les parcelles et la durée des plants en pépinière sont négativement corrélées à l'intensité des attaques de $H$. armigera et $T$. absoluta. Le nombre de traitements insecticides est négativement corrélé à l'infestation de T. absoluta. Le type de préparation du sol influe sur les infestations de T. absoluta. Les attaques de $H$. armigera varient en fonction du type d'irrigation. Les autres pratiques n'avaient pas d'effets sur les infestations de $T$. absoluta et $H$. armigera. Cette étude permet de connaître les relations entre les pratiques culturales et les insectes ravageurs de la tomate et peut ainsi renforcer les stratégies de gestion durable des bioagresseurs.

(C) 2016 International Formulae Group. All rights reserved.

Mots clés : Pratiques culturales, Helicoverpa armigera, Tuta absoluta, tomate, traitements insecticides, Sénégal.

\section{Cultural practices affect attacks of two pests of tomato in the Niayes in Senegal}

\begin{abstract}
Tomato crops are attacked by several insect pests including Helicoverpa armigera and Tuta absoluta. In order to assess the effect of crop management on these major pests in the Niayes (Senegal), a sample of 98
\end{abstract}


plots was monitored over four crop cycles in the dry season, from 2012 to 2014 . Cultural practices were collected by farmer interviews. Results showed that incidence of H. armigera and T. absoluta is negatively correlated with distance to the sea. Plant stand in plots and duration of plants in nursery were negatively correlated to infestation of $H$. armigera and T. absoluta. The number of insecticide applications was negatively correlated to the infestation of T. absoluta. The type of soil preparation influenced infestations of T. absoluta. Damage caused by $H$. armigera varied depending on the type of irrigation. Other practices had no effect on infestations of $T$. absoluta and $H$. armigera. This study provides information on the relationships between crop management and insect pests of tomato and can strengthen sustainable pest management strategies.

(c) 2016 International Formulae Group. All rights reserved.

Keywords: Cultural practices, Helicoverpa armigera, Tuta absoluta, tomato, insecticides, Senegal.

\section{INTRODUCTION}

Au Sénégal, la tomate est la deuxième spéculation marâichère la plus cultivée derrière l'oignon. Elle représente $22,53 \%$ de la production globale de légumes estimée, à 710000 tonnes (ANDS, 2013). Elle compte parmi les légumes les plus consommés (Huat et David-Benz, 2000).

Cette culture est cependant soumise à de nombreuses contraintes, dont la menace permanente des ravageurs (Mailafiya et al., 2014; Choudourou et al., 2012). Au Sénégal, deux Lépidoptères ; la "Noctuelle de la tomate", Helicoverpa armigera (Hübner); Noctuidae et la "mineuse sud-américaine", Tuta absoluta (Meyrick); Gelechiidae sont les ravageurs principaux de la tomate. Les dégâts des chenilles de la noctuelle peuvent atteindre 28\% (Diatte et al., 2015). Quant à T. absoluta, elle constitue une menace pour la production de tomates depuis sa détection en 2012 au Sénégal (Pfeiffer et al., 2013). T. absoluta est signalée partout dans les Niayes avec des pertes allant jusqu'à pousser à l'abandon des parcelles par les producteurs de la zone Sud (Brévault et al., 2014).

Les pratiques culturales sur la tomate peuvent influer sur la prolifération des insectes ravageurs. Les impacts de ces pratiques dans les systèmes maraîchers en Afrique sont peu connus. Les quelques études réalisées dans ce domaine portent sur les applications d'insecticides, les associations culturales, l'utilisation de fumure organique et leurs impacts sur les insectes ravageurs. Au Cameroun, l'utilisation abusive de matières actives a conduit à une perte de sensibilité des ravageurs (Achaleke et al., 2009; Achaleke et Brévault, 2010). Au Nigeria, l'application inappropriée d'insecticides ainsi qu'un mauvais dosage sont à la base des dégâts causés par les insectes. Il a été également constaté que l'infestation était réduite dans des champs où la tomate était associée avec des céréales, des tubercules ou d'autres légumes et que la plupart des pratiques n'avaient pas d'impact sur l'abondance des insectes ravageurs (Umeh et al., 2002). Au Sénégal, l'utilisation de la fumure organique de cheval et de poisson a entraîné une réduction des dégâts liés à $H$. armigera de $24 \%$, comparé à la fumure organique de mouton (Niassy et al., 2010).

Peu d'études sur l'impact des pratiques culturales sur l'infestation des ravageurs de la tomate ont été jusque-là menées au Sénégal. L'objectif de ce travail était d'évaluer l'influence des pratiques culturales sur les deux principaux insectes ravageurs de la tomate dans les Niayes au Sénégal. Il s'agissait de faire un échantillonnage d'insectes sur un réseau de 98 parcelles de 2012 à 2014 et de questionner les producteurs sur leurs pratiques culturales. 


\section{MATERIEL ET METHODES} Sites d'échantillonnages

Ce travail a été effectué dans la zone des Niayes au Sénégal. Cette zone s'étend sur une longueur de $180 \mathrm{~km}$ bordant la frange maritime du Nord du pays partant de Dakar à Saint-Louis en passant par la bordure Ouest de Thiès et de Louga. Sa largeur varie de cinq à $30 \mathrm{~km}$ à l'intérieur des terres. Elle constitue un milieu original caractérisé par des dunes et des dépressions souvent inondées par l'affleurement de la nappe phréatique et par un climat assez favorable aux cultures maraîchères. Inscrites dans la moitié Sud de la zone sahélienne, les Niayes sont caractérisées par l'alternance de deux saisons : une saison humide concentrée sur trois mois (juillet, août et septembre) et une saison sèche qui dure les autres neuf mois et durant laquelle se succède deux cycles de cultures maraîchères. Le climat chaud et sec, est caractérisé par la mousson qui souffle durant la saison humide. La pluviométrie est faible et dépasse rarement $450 \mathrm{~mm} / \mathrm{an}$. Les températures sont modérées et influencées par la circulation des alizés maritimes soufflés par les courants d'airs froids du nord (Açores). La température moyenne mensuelle la plus chaude oscille autour de $28{ }^{\circ} \mathrm{C}$ et la température minimale est inférieure à $18{ }^{\circ} \mathrm{C}$. Entre mai et juin, la présence de l'harmattan peut élever la température à un maximum de $31{ }^{\circ} \mathrm{C}$ (Diallo et al., 2015). Les sols varient du nord au sud. Leur nature et leur distribution s'articulent autour des grands ensembles géomorphologiques présents sur le territoire. On distingue ainsi six types de sols (Fall et al., 2001): les sols minéraux bruts d'apport qui caractérisent les dunes vives dans lesquelles l'évolution des sols y est pratiquement nulle; les sols ferrugineux tropicaux peu lessivés qui caractérisent les dunes rouges et occupant près de $70 \%$ de la région des Niayes; les sols brunrouges iso-humiques que l'on rencontre dans la partie nord-ouest de Louga et sud-ouest de
Saint-Louis; les vertisols rencontrés dans les dépressions à argile gonflante, localisées dans la zone de Sébikotane (plateau de Bargny), sur l'axe Somone et le lac Tanma; les sols halomorphes caractérisés par leur salinité et/ou alcalinité, situés aux environs des lagunes côtières barrées par les cordons dunaires et les sols hydromorphes des dépressions très favorables au développement du maraîchage.

Un suivi de 98 parcelles d'études a été effectué dans trois zones (Sud, Centre, Nord) des Niayes, le long d'un transect de Dakar Saint-Louis. Ce travail a été effectué sur quatre cycles de cultures, entre octobre 2012 et mai 2014. Les parcelles d'étude ont été géo référencées par GPS. Elles étaient distantes l'une de l'autre d'au moins deux kilomètres (Figure 1).

\section{Echantillonnages proprement dit}

L'échantillonnage se déroulait toutes les trois semaines dans chaque parcelle, depuis le repiquage jusqu'à la première date de récolte. Dans chaque parcelle, 24 plants identifiés aléatoirement sont observés. Trois feuilles situées à différents niveaux de la plante sont observées (une en haut, une au milieu et une en bas) pour calculer le pourcentage de feuille infesté par T. absoluta. Le nombre total de fruit et le nombre de fruit attaqués par $H$. armigera ont été également dénombrés afin d'évaluer l'incidence de ce ravageur.

\section{Enquêtes}

Parallèlement aux échantillonnages, les données sur les pratiques culturales (précédent cultural, travail du sol, type de sol, mode d'irrigation, variété, traitement insecticide, fertilisation, date de semis, date de repiquage, date de récolte, association culturale) de chaque parcelle sont notées. Elles ont été recueillies par questionnaire lors de chaque visite auprès des producteurs. 
Le calcul de la densité a été faite par l'intermédiaire d'un carré de densité, qui est un carré de $1 \mathrm{~m}^{2}$ posé au hasard dans la parcelle pour y effectuer un comptage de tous les plants et mauvaises herbes. Au total, 5 carrés de densité ont été posés dans chaque parcelle, ensuite le calcul de la moyenne a permis d'obtenir la densité de la parcelle.

\section{Analyse statistique des données}

Les données recueillies sont enregistrées dans un tableur Excel et analysées avec le logiciel Minitab 17. L'effet des pratiques sur les populations de ravageurs a été déterminé par une analyse de variance (ANOVA) et une corrélation. Une corrélation de Pearson a été utilisée au seuil de significativité de 5\%. Pour les ANOVA, les moyennes ont été séparées avec le test de Tukey $(\mathrm{P}<0,05)$. Préalablement aux analyses, une transformation de type $\log 10(\mathrm{x}+1)$ a été appliquée aux données pour garantir la normalité des distributions et l'égalité des variances.

\section{RESULTATS}

Les pratiques culturales ont une influence sur les deux principaux insectes ravageurs de la tomate dans les Niayes au Sénégal (Tableau 1 ; Figures 2 et 3).

L'incidence de $H$. armigera et de $T$. absoluta est négativement corrélée à la distance à la mer $(\mathrm{p}<0,01)$. La densité des plants dans les parcelles est négativement corrélée à l'intensité des attaques de $H$. armigera $(\mathrm{p}<0,05)$ et T. absoluta $(\mathrm{p}<0,01)$. La durée des plants en pépinière est négativement corrélée à l'intensité des attaques de $H$. armigera $(\mathrm{p}<0,05)$ et $T$. absoluta $(\mathrm{p}<0,01)$. Le nombre de traitements insecticides est négativement corrélé à l'infestation de $T$. absoluta ( $\mathrm{p}<0,01)$ (Tableau 1).

Le type d'irrigation des plants influe sur les infestations de $H$. armigera $(\mathrm{dl}=3$, $\mathrm{F}=3,22, \mathrm{P}<0,05)$. Les parcelles irriguées avec des aspersoirs $(0,08 \%)$, des lances $(0,04 \%)$, des arrosoirs et des seaux $(0,04 \%)$, ont été moins infestées par $H$. armigera que les autres parcelles irriguées par les techniques du goutte-à-goutte et de décrue $(0,09 \%)$ (Figure 2). Les infestations dues à $H$. armigera n'ont pas été influencées par le précédent cultural ( $\mathrm{dl}=5 ; \mathrm{F}=1,29, \mathrm{P}=0,273)$, le type de sol $(\mathrm{dl}=2$, $\mathrm{F}=0,23, \mathrm{P}=0,791$ ), le type de préparation $\mathrm{du}$ sol ( $d l=3, F=0,39, P=0,762)$, l'application ou non de fumure $(\mathrm{dl}=1, \mathrm{~F}=0,65, \mathrm{P}=0,424)$, la variété $(\mathrm{dl}=2, \mathrm{~F}=0,130, \mathrm{P}=0,88)$ (Figure 2).

Le type de préparation du sol influe sur les infestations de $T$. absoluta $(\mathrm{dl}=2, \mathrm{~F}=8,29$, $\mathrm{P}<0,01)$. Les infestations sont plus élevées dans les parcelles labourées à la « daba » (33\%) que dans celles qui sont hersées $(7 \%)$ ou travaillées mécaniquement (7\%) (Figure 3). Les infestations dues à $T$. absoluta n'ont pas été influencées par le précédent cultural $(\mathrm{dl}=5, \mathrm{~F}=0,58, \mathrm{P}=0,714)$, le type de sol (dl=2, $\mathrm{F}=0,91, \mathrm{P}=0,427)$, l'application ou non de fumure $(\mathrm{dl}=1, \mathrm{~F}=0,727, \mathrm{P}=0,41)$, le type d'irrigation $(\mathrm{dl}=4, \mathrm{~F}=1,57, \mathrm{P}=0,245)$ et la variété $(\mathrm{dl}=2, \mathrm{~F}=1,01, \mathrm{P}=0,406)$ (Figure 3). 

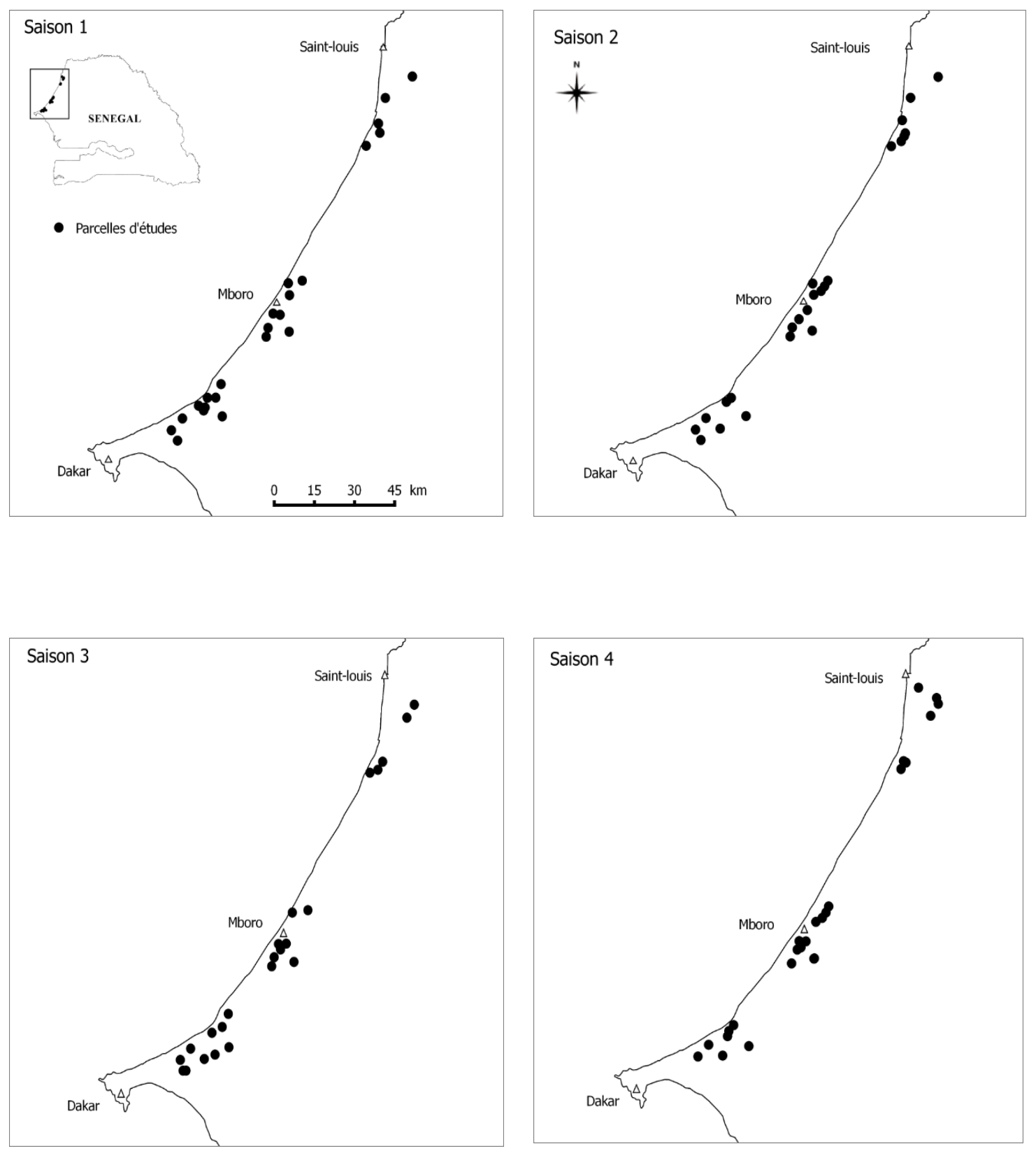

Figure 1 : Répartition géographique des parcelles d'étude dans la zone des Niayes. Phase 1: oct-12jan13, $n=24$ parcelles ; Phase 2 : fev13-mai-13n=26 parcelles ; Phase 3 : oct-13-jan-14, n=23 parcelles ; Phase 4 : fév.-14mai- $14, \mathrm{n}=25$ parcelles. 

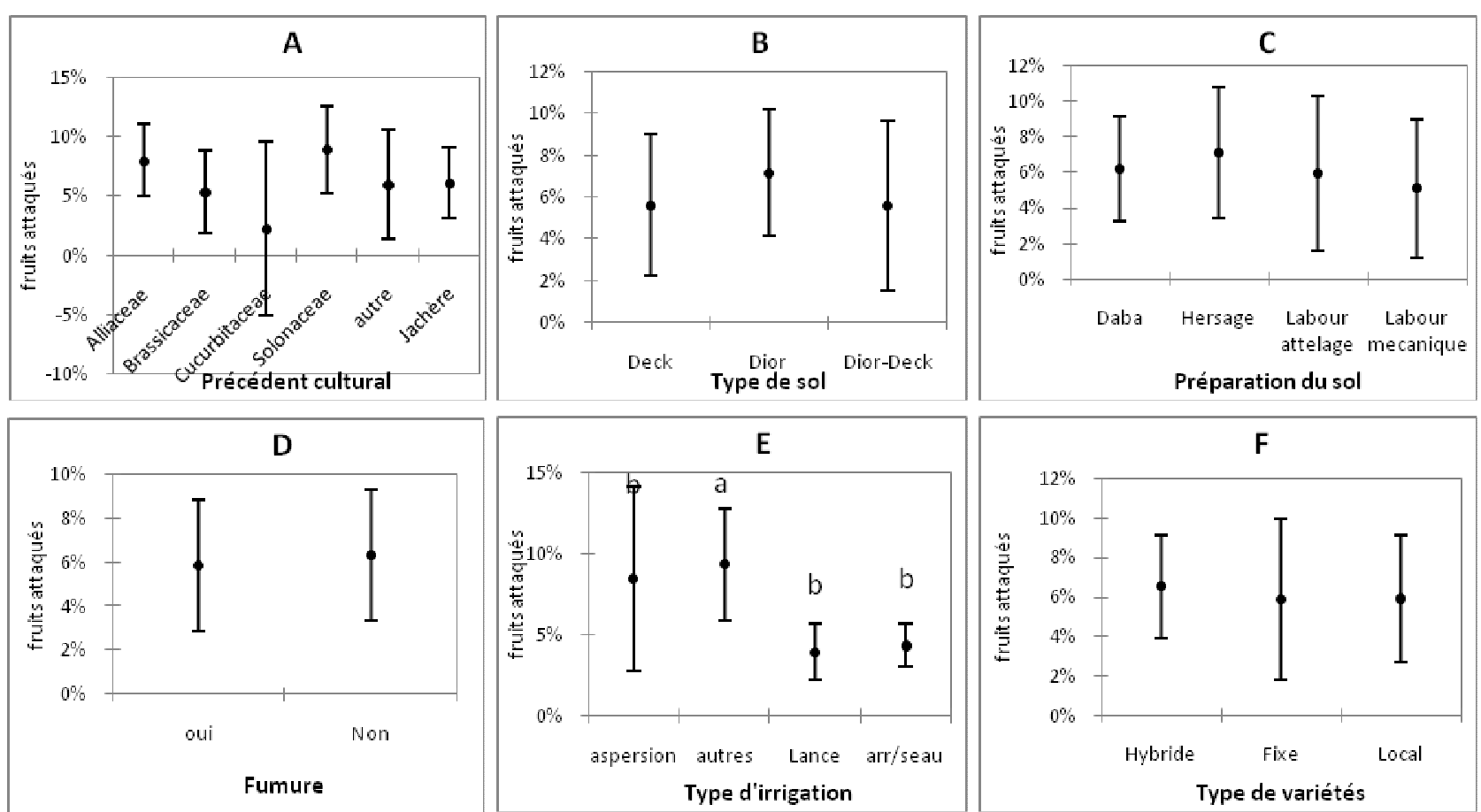

Figure 2 : Pourcentage de fruits attaqués par les larves de $H$. armigera selon les pratiques culturales. N= 98 parcelles ; A : précédent cultural, B : Type de sol, C : préparation du sol, $\mathrm{D}$ : fumure, E : type d'irrigation : (autres= décrue, goutte à goutte; arr/seau=arrosoir et seau), F : Type de variétés. (ANOVA, test de comparaison de Tuckey au seuil de $5 \%$ ). 

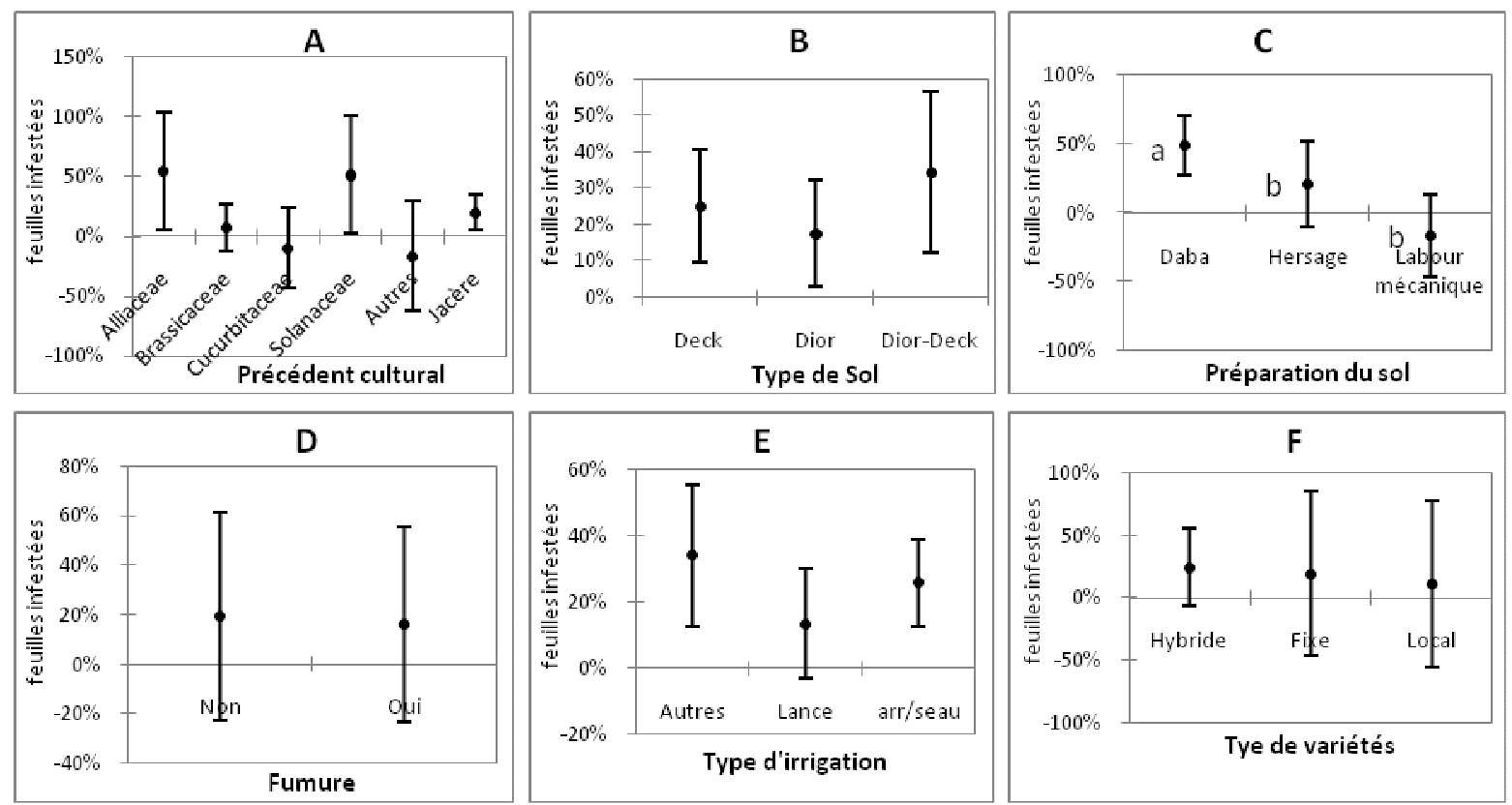

Figure 3 : Pourcentage de feuilles infestées par T. absoluta selon les pratiques culturales. N= 98 parcelles ; A : précédent cultural, B : Type de sol, C : préparation du sol, D : fumure, $\mathrm{E}:$ type d'irrigation : (autres= décrue, goutte à goutte ; arr/seau=arrosoir et seau), F : Type de variétés. (ANOVA, test de comparaison de Tuckey au seuil de $5 \%$ ). 
M. DIATTE et al. / Int. J. Biol. Chem. Sci. 10(2): 681-693, 2016

Tableau 1 : Interaction entre les pratiques culturales et les infestations par les deux principaux ravageurs de la tomate (H. armigera et T. absoluta) dans la zone des Niayes.

\begin{tabular}{|c|c|c|c|c|c|c|c|c|c|c|c|c|c|c|}
\hline Variables & $\%$ fruit $\mathrm{Ha}$ & \%_inf_Tuta & Di_mer & d_pl/ $/ \mathbf{m}^{2}$ & d_herbe/ $\mathrm{m}^{2}$ & Dr_Pep & fum_eq_N & urée & npk & Nb_tr_ins & tr_pep & Assoc & tr_bio & tr_bin \\
\hline$\%$ fruit $\mathrm{Ha}$ & 1 & 0,138 & $-0,301 * *$ & $-0,236^{*}$ & $-0,044$ & $-0,220 *$ & $-0,067$ & 0,150 & 0,032 & 0,146 & $-0,031$ & 0,006 & 0,097 & $-0,063$ \\
\hline$\%$ _inf_Tuta & 0,138 & 1 & $-0,342 * *$ & $-0,207 *$ & $-0,151$ & $-0,322 * *$ & $-0,157$ & $-0,195$ & $-0,135$ & $-0,316^{* *}$ & $-0,167$ & 0,105 & 0,042 & $-0,194$ \\
\hline Di_sea & $-0,301 * *$ & $-0,342 * *$ & 1 & $0,378 * *$ & $0,291 * *$ & $0,706 * * *$ & 0,087 & 0,042 & 0,135 & 0,141 & 0,032 & $-0,062$ & $-0,001$ & $-0,001$ \\
\hline d_pl/m2 & $-0,236^{*}$ & $-0,207^{*}$ & $0,378 * * *$ & 1 & 0,163 & $0,340 * *$ & 0,028 & $-0,066$ & $-0,261^{*}$ & $0,250 *$ & $-0,099$ & $-0,119$ & $-0,175$ & $-0,014$ \\
\hline d_herbe & $-0,044$ & $-0,151$ & $0,291 * *$ & 0,163 & 1 & $0,294 * *$ & 0,087 & $-0,116$ & 0,162 & 0,072 & 0,049 & 0,161 & 0,134 & $-0,130$ \\
\hline Dr_Pep & $-0,220^{*}$ & $-0,322 * *$ & $0,706^{* * *}$ & $0,340 * *$ & $0,294 * *$ & 1 & 0,054 & 0,137 & 0,063 & $0,210^{*}$ & 0,006 & 0,040 & 0,049 & 0,039 \\
\hline fum_eq_N & $-0,067$ & $-0,157$ & 0,087 & 0,028 & 0,087 & 0,054 & 1 & 0,044 & 0,108 & 0,096 & $0,297 * *$ & 0,049 & $-0,069$ & 0,121 \\
\hline Urée & 0,150 & $-0,195$ & 0,042 & $-0,066$ & $-0,116$ & 0,137 & 0,044 & 1 & 0,056 & $0,438 * * *$ & 0,146 & $0,203^{*}$ & 0,028 & 0,140 \\
\hline Npk & 0,032 & $-0,135$ & 0,135 & $-0,261^{*}$ & 0,162 & 0,063 & 0,108 & 0,056 & 1 & 0,093 & 0,117 & 0,105 & 0,166 & 0,073 \\
\hline Nb_tr_ins & 0,146 & $-0,316^{* *}$ & 0,141 & $0,250^{*}$ & 0,072 & $0,210^{*}$ & 0,096 & $0,438 * * *$ & 0,093 & 1 & $0,297 * *$ & $0,228 *$ & 0,083 & 0,176 \\
\hline tr_pep & $-0,031$ & $-0,167$ & 0,032 & $-0,099$ & 0,049 & 0,006 & $0,297 * *$ & 0,146 & 0,117 & $0,297 * *$ & 1 & 0,131 & 0,030 & $0,204^{*}$ \\
\hline Assoc & 0,006 & 0,105 & $-0,062$ & $-0,119$ & 0,161 & 0,040 & 0,049 & $0,203^{*}$ & 0,105 & $0,228^{*}$ & 0,131 & 1 & 0,106 & $-0,079$ \\
\hline Nb_tr_bio & 0,097 & 0,042 & $-0,001$ & $-0,175$ & 0,134 & 0,049 & $-0,069$ & 0,028 & 0,166 & 0,083 & 0,030 & 0,106 & 1 & $-0,123$ \\
\hline Nb_tr_bin & $-0,063$ & $-0,194$ & $-0,001$ & $-0,014$ & $-0,130$ & 0,039 & 0,121 & 0,140 & 0,073 & 0,176 & $0,204^{*}$ & $-0,079$ & $-0,123$ & 1 \\
\hline
\end{tabular}

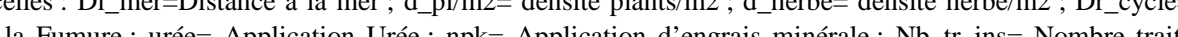

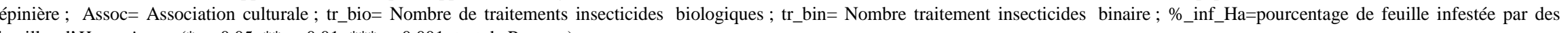
chenilles d'H. armigera. ( $\mathrm{p} p<0,05,{ }^{* *} \mathrm{p}<0,01,{ }^{* * *} \mathrm{p}<0,001$; test de Pearson). 


\section{DISCUSSION}

Les résultats de la présente étude révèlent que les pratiques culturales influencent les attaques des ravageurs. L'incidence de $H$. armigera ( 0 et $29 \%$ de fruits) et de $T$. absoluta ( 0 et $60 \%$ de feuilles infestées), est plus élevée au niveau des parcelles situées au bord de la mer. Au niveau du littoral, notamment entre Saint-Louis et Dakar, les alizés qui soufflent le long de la côte provoquent une baisse des températures avec une moyenne des maximales diurnes qui est de $24{ }^{\circ} \mathrm{C}$ de janvier à mars et entre 25 et 27 ${ }^{\circ} \mathrm{C}$ en avril, mai et décembre. Ces faibles températures sont propices au développement de $T$. absoluta, qui est un ravageur invasif, originaire des zones tempérées (Europe, méditerranéen) où il provoque d'importants dégâts allant jusqu'à $27 \%$ dans les cultures de tomate (Desneux et al., 2010; Desneux et al., 2011). La vitesse de développement de $T$. absoluta augmente lorsque la température baisse. Il est de 21,1 jours à $26{ }^{\circ} \mathrm{C}$ et 29,4 jours à $23,4{ }^{\circ} \mathrm{C}$ (Boualem et al., 2012). Cela est à l'origine de plusieurs dégâts sur tomate dans les Niayes (60\% de feuilles infestées) (Sylla et al., 2016). H. armigera est un ravageur qui évolue dans les zones plus chaudes avec un optimum de développement compris entre $25{ }^{\circ} \mathrm{C}$ et $27{ }^{\circ} \mathrm{C}$ (Mironidis et Savopoulou-Soultani, 2008). Sa présence en bordure des côtes pourrait s'expliquer par la disponibilité de la ressource. En effet, la tomate est une plante des basses températures avec un optimum de croissance qui se situe entre $21^{\circ} \mathrm{C}$ et $24^{\circ} \mathrm{C}$ (Naika et al., 2005).

La densité des plants dans les parcelles compris entre 2 et 20 plants $/ \mathrm{m}^{2}$ est négativement corrélée à l'intensité des attaques de $H$. armigera et $T$. absoluta. La forte densité des plants de tomate favoriserait le développement des ennemis naturels. Les plants de tomate constitueraient un habitat et un abri qui va favoriser et améliorer la régulation naturelle par les auxiliaires.
Toutefois, il a été montré que les paysages diversifiés détiennent plus de potentiel pour la conservation de la biodiversité et le maintien de la fonction de contrôle des ravageurs (Landis et al., 2000; Bianchi et al., 2006; Chaplin-Kramer et al., 2011; Chaplin-Kramer et al., 2013).

La durée des plants en pépinière est négativement corrélée à l'intensité des attaques de $H$. armigera et $T$. absoluta. Les plants repiqués sont en effet plus vigoureux à 30 jours de pépinière, donc plus résistants aux attaques des bioagresseurs. La production de plants sains et vigoureux pourrait être une méthode efficace pour renforcer les stratégies de gestion des ravageurs.

Le nombre de traitements insecticides compris entre 0 et 11 traitements par cycle de culture est négativement corrélé à l'infestation de $T$. absoluta. Ce résultat montre que les insecticides sont efficaces contre la mineuse de la tomate. En fait, T. absoluta est un nouveau ravageur qui vient d'être signalé dans les périmètres maraîchers des Niayes avec des dégâts allant jusqu'à 55\% de feuilles minées en zone Sud (Pfeiffer et al., 2013; Brévault et al., 2014). Sa gestion est faite par des insecticides chimiques de diverses familles dont les plus importantes sont les organophosphorés, les pyréthrinoïdes et les avermectines (Diatte et al., 2015). Les populations du ravageur n'ont pas encore développé une résistance par rapport aux insecticides contrairement au Brésil (Siqueira et al., 2000) et en Argentine (Lietti et al., 2005) où des formes de résistance ont été signalées. Aucune corrélation n'est observée entre le nombre de traitements insecticides et les attaques de $H$. armigera. Ce phénomène pourrait s'expliquer par une absence d'efficacité des produits chimiques contre la noctuelle de la tomate. En effet, $H$. armigera est à l'heure actuelle insensible à plusieurs familles d'insecticides à travers le monde. Sa résistance est signalée en Asie (Aheer, et al., 
2009), en Europe (Mironidiset al., 2013), en Afrique centrale (Achaleke et Brévault, 2010 ; Achaleke et al., 2009) en Afrique occidentale (Martin et al., 2000 ; Martin et al., 2002). Au Sénégal, $H$. armigera serait insensible aux pyréthrinoïdes et aux organophosphorés dans certaines zones des Niayes et de la vallée du Fleuve Sénégal (Brévault, communication personnelle).

Les attaques des fruits par les larves de H. armigera ont été influencées par le type d'irrigation. Les parcelles irriguées avec des aspersoirs, des lances, des arrosoirs et des seaux ont été moins infestées par $H$. armigera que les autres parcelles irriguées par les techniques du goutte-à-goutte et de décrue. En effet, les techniques d'aspersion lessiveraient les plants, entrainant la disparition des chenilles néonates et des œufs. Cette action est favorisée par le fait que les œufs sont déposés de préférence sur les feuilles les plus hautes (Mabbett et Nachapong, 1983; Firempong et Zalucki, 1990).

Le type de préparation du sol influe sur les infestations de $T$. absoluta. Les infestations sont plus élevées dans les parcelles labourées à la «daba » que dans celles qui sont hersées ou travaillées mécaniquement. Ceci pourrait être dû au travail profond du sol qui serait à l'origine de la destruction des chrysalides de $T$. absoluta qui se développent en partie dans le sol (Torres et al., 2001).

\section{Conclusion}

Ce travail a permis de montrer que les pratiques culturales ont une influence sur les deux principaux insectes ravageurs de la tomate dans les Niayes au Sénégal. L'incidence de $H$. armigera et de $T$. absoluta est plus élevée au niveau des côtes. Plus la densité des plants dans les parcelles est élevée, moins il y a d'attaques dues à $H$. armigera et $T$. absoluta. Plus la vigueur des plants en pépinières est grande, moins il y a d'infestation liée à H. armigera et T. absoluta. L'infestation due à $T$. absoluta est contrôlée par les traitements insecticides utilisés dans la zone. Le type d'irrigation du sol influe sur les infestations de $H$. armigera. Les techniques d'irrigation utilisant les aspersoirs, les lances, les arrosoirs et les seaux défavorisent la prolifération de $H$. armigera. Le type de préparation du sol influe sur les infestations de T. absoluta. Les infestations sont plus élevées dans les parcelles labourées à la «daba » que dans celles qui sont hersées ou travaillées mécaniquement. Des études approfondies sont nécessaires pour affiner les interactions qui existent entre les différents paramètres explicatifs. Cette étude permettra un renforcement des stratégies de gestion durable des ravageurs de la tomate en tenant compte des pratiques culturales qui influent sur la prolifération des ravageurs.

\section{REMERCIEMENTS}

Nous remercions profondément les personnes et les organismes qui sont intervenus lors de cette étude. Il s'agit particulièrement de $\mathrm{O}$. Ndoye et $\mathrm{S}$. Gueye (FPMN, Fédération des Producteurs Maraîchers de la zone des Niayes), et des étudiants en masters et doctorats de l'équipe 2PIA de l'UCAD pour l'aide apportée lors des prélèvements sur le terrain. Nous exprimons nos sincères gratitudes à E.V. Coly (DPV, Sénégal), pour la facilitation apportée dans le déroulement de ce travail.

\section{CONFLIT D'INTÉRÊT}

Nous déclarons qu'aucun conflit d'intérêt n'existe entre les auteurs. L'ordre des auteurs au niveau de l'article et le contenu du document a été validé dans l'unanimité.

\section{CONTRIBUTIONS DES AUTEURS}

MD est l'investigateur principal ; TB et DS-S ont co-encadré les travaux les travaux de terrain et ont participé à la rédaction du 
manuscrit; KD est l'initiateur du projet. Il a défini les protocoles et coordonné toutes les activités.

\section{RÉFÉRENCES}

Achaleke J, Brévault T. 2010. Inheritance and stability of pyrethroid resistance in the cotton bollworm Helicoverpa armigera (Lepidoptera: Noctuidae) in Central Africa. Pest Manag. Sci., 66(2): 137-141. DOI: http://dx.doi.org/10.1002/ps.1843

Achaleke J, Martin T, Ghogomu RT, Vaissayre M, Brévault T. 2009. Esterasemediated resistance to pyrethroids in field populations of Helicoverpa armigera (Lepidoptera: Noctuidae) from Central Africa. Pest Manag. Sci., 65(10):11471154. DOI: http://dx.doi.org/ 10.1002/ps. 1807

ANDS (Agence Nationale de la Démographie et de la Statistique). 2013. Situation Économique et Sociale Du Sénégal En 2011. ANDS ; 109p.

Aheer GM, Aziz MA, Hameed A, Ali A. 2009. Evaluation of resistance to different insecticides in field strains of Helicoverpa armigera (Lepidoptera: Noctuidae) in Punjab, Pakistan. Entomol. Res. 39(3):159-167. DOI: http://dx.doi.org/10.1111/j.17485967.2009.00210.x

Bianchi F, Booij CJH, Tscharntke T. 2006. Sustainable pest regulation in agricultural landscapes: a review on landscape composition, biodiversity and natural pest control. Proc. Roy. Soc. B: Bio. Sci., 273(1595): $\quad$ 1715-1727. DOI: http://dx.doi.org/10.1098/rspb.2006.3530

Boualem M, Allaoui H, Hamadi R, Medjahed M. 2012. Biologie et complexe des ennemis naturels de Tuta absoluta à Mostaganem (Algérie). EPPO Bull., 42(2): 268-274. DOI: http://dx.doi.org/ 10.1111/epp. 2570
Brévault T, Sylla S, Diatte M, Bernadas G, Diarra K. 2014. Tuta absoluta Meyrick (Lepidoptera: Gelechiidae): A New Threat to Tomato Production in SubSaharan Africa. Afr. Entomol., 22(2): 441-444. DOI: http://dx.doi.org/ 10.4001/003.022.0202

Chaplin-Kramer R, de Valpine P, Mills NJ, Kremen C. 2013. Detecting pest control services across spatial and temporal scales. Agric. Ecosyst. Environ., 181: 206-212. DOI: http://dx.doi.org/ 10.1016/j.agee.2013.10.007

Chaplin-Kramer R, O'Rourke ME, Blitzer EJ, Kremen C. 2011. A meta-analysis of crop pest and natural enemy response to landscape complexity. Ecol. Lett., 14(9):922-932. DOI: http://dx.doi.org/ 10.1111/j.1461-0248.2011.01642.x

Choudourou DC, Agbaka A, Adjakpa JB, Koutchika RE, Adjalian EJN. 2012. Inventaire préliminaire de l'entomofaune des champs de tomates (Lycopersicon esculentum Mill) dans la Commune de Djakotomey au Bénin. Int. J. Biol. Chem. Sci., 6(4):1798-1804. DOI: http://dx.doi.org/10.4314/ijbcs.v6i4.34

Desneux N, Luna MG, Guillemaud T, Urbaneja A, 2011. The invasive South American tomato pinworm, Tuta absoluta, continues to spread in AfroEurasia and beyond: the new threat to tomato world production. J. Pest Sci., 84: 403-408. DOI: http://dx.doi.org/ 10.1007/s10340-011-0398-6

Desneux N, Wajnberg E, Wyckhuys KAG, Burgio G, Arpaia S, Narvaez-Vasquez CA, Gonzalez-Cabrera J, Ruescas DC, Tabone E, Frandon J, Pizzol J, Poncet C, Cabello T, Urbaneja A. 2010. Biological invasion of European tomato crops by Tuta absoluta: ecology, geographic expansion and prospects for biological control. J. Pest Sci., 83:197-215. DOI: 
http://dx.doi.org/10.1007/s10340-0100321-6

Diallo MD, Ngamb T, Tine AK, Guisse M, Ndiaye O, Mahamat Saleh M, Diallo A, Seck S, Diop A, et al. 2015. Caractérisation agropédologique des sols de mboltime dans la zone des Niayes (Sénégal). Agronomie Africaine, 27(1):57-67.

http://www.ajol.info/index.php/aga/article /view/118754

Diatte M, Brévault T, Sylla S, Tendeng E, Sall-Sy D, Diarra K. 2015. New insect pest assemblage threatens field-grown tomato production in Senegal. Int. J. Tropic. Ins. Sci. (In press).

Fall AS, Fall ST, Cisse I, Badiane AN, Diao MB, Fall CA. 2001. Caractérisation de la zone des Niayes. In CITES Horticoles en Sursis? L'Agriculture Urbaine les Grandes Niayes au Sénégal. IDRC. http//idrc.ca/en/ev-27906-201-1 DO_TOPIC.htm.

Firempong S, Zalucki MP. 1990. Host PlantSelection by Helicoverpa armigera (Hubner) (Lepidoptera, Noctuidae)-Role of Certain Plant Attributes. Aust. J. Zool, 37(6): 675-683. DOI: http://dx.doi.org/ 10.1071/ZO9890675

Landis DA, Wratten SD, Gurr GM. 2000. Habitat management to conserve natural enemies of arthropod pests in agriculture. Annu. Rev. Entomol, 45(1): 175-201. DOI: http://dx.doi.org/10.1146/ annurev.ento.45.1.175

Lietti MM, Botto E, Alzogaray RA. 2005. Insecticide resistance in argentine populations of Tuta absoluta (Meyrick)(Lepidoptera: Gelechiidae). Neotrop. Entomol., 34(1): 113-119. DOI: http://dx.doi.org/10.1590/S1519566X2005000100016

Mabbett TH, Nachapong M. 1983. Some aspects of oviposition by Heliothis armigera pertinent to cotton pest management in Thailand. Int. J. Pest Manag, 29(2): 159-165. DOI: http://dx.doi.org/10.1080/0967087830937 0792

Mailafiya DM, Degr MM, Maina YT, Gadzama UN, Galadima IB. 2014. Preliminary Studies on Insect Pest Incidence on Tomato in Bama, Borno State, Nigeria. Int. Let. Nat. Scie, 5: 4554.

Martin T, Chandre F, Ochou OG, Vaissayre M, Fournier D. 2002. Pyrethroid resistance mechanisms in the cotton bollworm Helicoverpa armigera (Lepidoptera: Noctuidae) from West Africa. Pesticide Biochemistry and Physiology, 74(1): 17-26. DOI: doi:10.1016/S0048-3575(02)00117-7

Martin T, Ochou GO, Hala-N'Klo F, Vassal JM, Vaissayre M. 2000. Pyrethroid resistance in the cotton bollworm, Helicoverpa armigera (Hübner), in West Africa. Pest Management Science, 56(6): 549-554. DOI: 10.1002/(SICI)15264998(200006).

Mironidis GK, Kapantaidaki D, Bentila M, Morou E, Savopoulou-Soultani M, Vontas J. 2013. Resurgence of the cotton bollworm Helicoverpa armigera in northern Greece associated with insecticide resistance. Insect Sci., 20(4): 505-512. DOI: http://dx.doi.org/ 10.1111/j.1744-7917.2012.01528.x

Mironidis GK, Savopoulou-Soultani M. 2008. Development, Survivorship, and Reproduction of Helicoverpa armigera (Lepidoptera: Noctuidae) Under Constant and Alternating Temperatures. Environ. Entomol., 37(1): 16-28. DOI: http://dx.doi.org/ 10.1093/ee/37.1.16

Naika S, de Jeude J van L, de Goffau M, Hilmi M, Van Dam B. 2005. La Culture de la Tomate: Production, Transformation et Commercialisation 
( $5^{\text {eme }}$ edn). Digigrafi: Wageningen, PaysBas; 105.

Niassy S, Diarra K, Niang Y, Niang S, Pfeifer HR. 2010. Effect of organic fertilizers on the susceptibility of tomato Lycopersicon esculentum: Solanaceae to Helicoverpa armigera Lepidoptera: Noctuidae in the Niayes area Senegal. Res. J. Agric. Biol. Sci., 6(6): 708-12. URL: http://www.cabdirect.org/abstracts/20113 079800.html

Pfeiffer DG, Muniappan R, Sall D, Diatta P, Diongue A, Dieng EO. 2013. First Record of Tuta absoluta (Lepidoptera: Gelechiidae) in Senegal. Fla. Entomol., 96(2): 661-662. DOI: http://dx.doi.org/ 10.1653/024.096.0241

Siqueira HÁA, Guedes RNC, Picanço MC. 2000. Insecticide resistance in populations of Tuta absoluta (Lepidoptera: Gelechiidae). Agric. For. Entomol., 2(2): 147-153. DOI: http://dx.doi.org/10.1046/j.14619563.2000.00062.x/abstract

Sylla S, Brévault T, Bal AB, Tendeng E, Sow A, Diatte M, Desneux N, Diarra K. 2016. Rapid spread and damage to tomato crops of the invasive pest, Tuta absoluta (Lepidoptera, Gelechiidae), in Senegal. Plos one., (In press).
Torres JB, Faria CA, Jr WSE, Pratissoli D. 2001. Within-plant distribution of the leaf miner Tuta absoluta (Meyrick) immatures in processing tomatoes, with notes on plant phenology. Int. J. Pest Manag., 47(3): 173-78. DOI: http://dx.doi.org/ 10.1080/02670870010011091

Umeh VC, Kuku FO, Nwanguma EI, Adebayo OS, Manga AA. 2002. A survey of the insect pests and farmers' practices in the cropping of tomato in Nigeria. Tropicultura. Trimestriel (octobre novembre - décembre), avec le soutien de la Direction Générale de la Coopération Internationale

(DGCI).

Verantwoordelijke uitgever: J.

Vercruysse, 181-186.

Huat J, David-Benz H. 2000. La tomate d'industrie au Sénégal: performances de la production et enjeux pour la filière. Legoupil JC Dancette C Godon P Maïga IM Ndiaye KM Ed Pour Un Dév. Durable Agric. Irriguée Dans Zone SoudanoSahél. PSIWECARD-CORAF. 2000: 167187. URL: http://publications.cirad. fr/une_notice.php?dk=477825. 